\title{
Study on differentiability problems of interval-valued functions
}

\author{
Yu-E Bao ${ }^{\mathrm{a}, *}$, Jin-Jun Li ${ }^{\mathrm{a}}$, Eer-Dun Bai ${ }^{\mathrm{b}}$ \\ ${ }^{a}$ College of Mathematics, Inner Mongolia University for Nationalities, Tongliao, Inner Mongolia 028043, P. R. China. \\ ${ }^{b}$ College of Computer Science and Technology, Inner Mongolia Universities, Tongliao, Inner Mongolia 028043, P. R. China. \\ Communicated by V. K. Le
}

\begin{abstract}
In this paper, we give the concepts of H-directional differentiability and D-directional differentiability of interval-valued functions. Then we discuss the properties of $\mathrm{H}$-directional differentiable interval-valued functions and D-directional differentiable interval-valued functions. The necessary and sufficient conditions for the H-directional differentiability are given together with the sufficient conditions and the necessary and sufficient conditions for D-directional differentiability of interval-valued functions. Then we discuss the relationship between the two directional differentiability and prove these directional differentiability can be equivalent under a certain conditions. (C)2017 All rights reserved.
\end{abstract}

Keywords: Hukuhara difference, Hausdorff distance, interval-valued function, H-directional differentiability, D-directional differentiability.

2010 MSC: 46T20, 46G05.

\section{Introduction}

Interval analysis (or interval mathematics), whose basic idea is to use interval variables instead of point variables in calculation, was firstly formulated by Moore [4] in 1966. From then on, the theory of interval analysis and its application developed greatly with the joint effort of many researchers. Especially, in order to build the theory of optimization of interval value $[5,7,8]$, theory of differential equation of interval value $[1,2,6]$ and the differential theory of fuzzy value [3], several kinds of differentiability of interval-valued functions were invented, and the related theories were built.

Generally speaking, there are two different ways to define the differentiability of the interval-valued function. The first one is to use the concepts of $\mathrm{H}$-derivative of interval-valued function from nonempty subset of real number space to interval number space, and the partial $\mathrm{H}$-derivative of interval-valued function from nonempty subset of $n$-dimensional Euclidean space to interval number space, which are given by $\mathrm{H}$-difference $[1,7,8]$. The second one is to use the concepts of $\mathrm{gH}$-derivative of interval-valued function from nonempty subset of real number space to interval number space, and the partial $\mathrm{gH}$ derivative of interval-valued function from nonempty subset of $n$-dimensional Euclidean space to interval number space, which are given by gH-difference $[2,3,5]$. Only the changing rate of interval-valued function in axis direction is taken into consideration, rather than in other special directions.

\footnotetext{
*Corresponding author

Email address: byebed@163.com (Yu-E Bao)
} 
In this paper, we study the changing rate of interval-valued function which is from nonempty subset of $n$-dimensional Euclidean space into interval number space in special direction, i.e., $\mathrm{H}$-directional differentiability and D-directional differentiability problem, which enrich and develop the theory of differential of interval-valued function and its application. In Section 3, we build the concepts of H-directional differentiability and $\mathrm{H}$-directional derivative, and prove that $\mathrm{H}$-derivative and partial $\mathrm{H}$-derivative are both directional derivative of interval-valued function in axis direction. In Section 4, we build the concepts of D-directional differentiability and D-directional partial derivative. And we study the relation between $\mathrm{H}$-directional differentiability and D-directional differentiability. So that we can prove that an intervalvalued function which is $\mathrm{H}$-differentiable must be $\mathrm{D}$-differentiable, but the opposite is not always true.

\section{Basic concepts of interval number}

Let $R$ be the real line. For $\underline{a}, \bar{a} \in R$ and $\underline{a} \leqslant \bar{a}$, we call the bounded closed interval $[\underline{a}, \bar{a}]$ an interval number, and denote $a=[a, \bar{a}]$. The set of all interval numbers of real line $R$ are denoted as $[R]$, which is called interval number space. Let $M$ be a nonempty subset of an $n$-dimensional Euclid space $R^{n}$. Let $F: M \rightarrow[R]$ be an interval-valued function $F(x)=[\underline{F}(x), \bar{F}(x)]$, which $\underline{F}(x)$ and $\bar{F}(x)$ are real-valued functions defined on $M$ satisfying $\underline{F}(x) \leqslant \bar{F}(x)$ for any $x \in M$. In this paper, for $x \in M, y \in R^{n}$, the directional derivatives of $\underline{F}(x)$ and $\bar{F}(x)$ in direction $y$ at $x$ denotes as $\underline{F}(x, y)$ and $\bar{F}(x, y)$, respectively.

For $\mathrm{a}=[\underline{\mathrm{a}}, \overline{\mathrm{a}}], \mathrm{b}=[\underline{\mathrm{b}}, \overline{\bar{b}}] \in[\mathrm{R}]$ and $\mathrm{k} \in \mathrm{R}$, we define the operations of addition and scalar multiplication of interval numbers by

$$
a+b=[\underline{a+b}, \overline{a+b}]=[\underline{a}+\underline{b}, \bar{a}+\bar{b}],
$$

and

$$
k a=[k a, \overline{k a}]= \begin{cases}{[k \underline{a}, k \bar{a}],} & k \geqslant 0, \\ {[k \bar{a}, k \underline{a}],} & k<0 .\end{cases}
$$

The metric structure is usually given by the Hausdorff distance between interval numbers

$$
\mathrm{D}_{\mathrm{H}}(\mathrm{a}, \mathrm{b})=\max \{|\underline{a}-\underline{b}|,|\overline{\mathrm{a}}-\overline{\mathrm{b}}|\},
$$

where $a=[\underline{a}, \bar{a}], b=[\underline{b}, \bar{b}]$. Then $\left([R], D_{H}\right)$ is a complete metric space, and for $a, b, c \in[R]$ and $k \in R$ there are following properties:

$$
D_{H}(a+c, b+c)=D_{H}(a, b), \quad D_{H}(k a, k b)=|k| D_{H}(a, b) .
$$

Definition 2.1 ([7]). Let $a, b \in[R]$. If there exists $c \in[R]$ such that $a=b+c$, then $c$ is called the Hukuhara difference (H-difference), which is denoted as $c=a-H b$.

By Definition 2.1 we can easily obtain the following properties.

Proposition 2.2. Let $\mathrm{a}, \mathrm{b} \in[\mathrm{R}]$. If $\mathrm{H}$-difference $\mathrm{a}-{ }_{\mathrm{H}} \mathrm{b}$ exists, i.e., $\mathrm{c}=\mathrm{a}-\mathrm{H} \mathrm{b} \in[\mathrm{R}]$, then

$$
\overline{\mathrm{c}}=\overline{\mathrm{a}}-\overline{\mathrm{b}} \text { and } \underline{\mathrm{c}}=\underline{\mathrm{a}}-\underline{\mathrm{b}} .
$$

Proposition 2.3. Let $\mathrm{a}, \mathrm{b} \in[\mathrm{R}]$, and $\mathrm{H}$-difference exists, then

$$
D_{H}(a, b+c)=D_{H}(a-H b, c) .
$$

Proof. By the Hausdorff distance equation and Proposition 2.2, we can easily obtain it.

Definition 2.4 ([7]). Let $M$ be an open set in R. An interval-valued $F: M \rightarrow\left([R], D_{H}\right)$ is $H$-differentiable at $x^{0} \in M$ if there exists an $F_{H}^{\prime}\left(x^{0}\right) \in[R]$ such that

$$
\lim _{h \rightarrow 0^{+}} \frac{F\left(x^{0}+h\right)-{ }_{H} F\left(x^{0}\right)}{h}=\lim _{h \rightarrow 0^{+}} \frac{F\left(x^{0}\right)-{ }_{H} F\left(x^{0}-h\right)}{h}=F_{H}^{\prime}\left(x^{0}\right) .
$$

In this case, $F_{H}^{\prime}\left(x^{0}\right)$ is called the $H$-derivative of $F$ at $\chi^{0}$. 
Definition 2.5 ([7]). Let $F: M \rightarrow\left([R], D_{H}\right)$ be an interval-valued function and $x^{0}=\left(x_{1}^{0}, \cdots, x_{n}^{0}\right) \in M$. If interval-valued function

$$
h_{i}\left(x_{i}\right)=F\left(x_{1}^{0}, \cdots, x_{i-1}^{0}, x_{i}, x_{i+1}^{0}, \cdots, x_{n}^{0}\right),
$$

H-differentiable at $x_{i}^{0}$, we say that $F(x)$ has the $i$-th partial $H$-derivative $F$ of at $x^{0}$. We can denote $h_{i}^{\prime}\left(x_{i}^{0}\right)$ as $\frac{\partial F}{\partial x_{i}}\left(x^{0}\right)$, i.e., $\frac{\partial F}{\partial x_{i}}\left(x^{0}\right)=h_{i}^{\prime}\left(x_{i}^{0}\right) \in[R]$.

\section{H-Directional differentiability of interval-function}

In $([7,8])$, Wu defined the partial $\mathrm{H}$-derivative and $\mathrm{H}$-differentiability of interval-valued function from a subset of space $R^{n}$ into interval number space $[R]$ by using $\mathrm{H}$-difference of interval numbers. In the following, by using $\mathrm{H}$-difference of interval numbers, we define the $\mathrm{H}$-directional derivative and $\mathrm{H}$ directional differentiability of interval-valued function from subset of space $R^{\mathfrak{n}}$ to interval number space [R].

In this paper, for $y \in R^{n}$, the unit vector of $y$ is denoted as $y_{e}$.

Definition 3.1. Let $F: M \rightarrow\left([R], D_{H}\right)$ be an interval-valued function and $x \in M$. If for $y \in R^{n}$, there exists $\delta>0$ such that $x+h y_{e} \in M\left(x-h y_{e} \in M\right)$ and the H-difference

$$
F\left(x+h y_{e}\right)-{ }_{H} F(x)\left(F(x)-{ }_{H} F\left(x-h y_{e}\right)\right),
$$

exists for any real number $h \in(0, \delta)$, and there exists $a_{+} \in[R]\left(a_{-} \in[R]\right)$ such that

$$
\lim _{h \rightarrow 0^{+}} \frac{1}{h}\left[F\left(x+h y_{e}\right)-_{H} F(x)\right]=a^{+}\left(\lim _{h \rightarrow 0^{+}} \frac{1}{h}\left[F(x)-{ }_{H} F\left(x-h y_{e}\right)\right]=a^{-}\right) .
$$

Then we say that $F$ is right (resp. left) $H$-differentiable in the direction $y$ at $x, a_{+}\left(a_{-}\right)$is the right (resp. left) directional derivative of $F$ in direction $y$ at $x$, and denote by

$$
\mathrm{F}_{+}^{\mathrm{H}}(x, y)=a_{+}\left(F_{-}^{\mathrm{H}}(x, y)=a_{-}\right) .
$$

If $\mathrm{F}_{+}^{\mathrm{H}}(x, y)=\mathrm{F}_{-}^{\mathrm{H}}(x, y)$, we say that $\mathrm{F}$ is $\mathrm{H}$-differentiable in direction $\mathrm{y}$ at $\mathrm{x}$, which is denoted as

$$
\mathrm{F}^{\mathrm{H}}(x, y)=\mathrm{F}_{+}^{\mathrm{H}}(x, y)=\mathrm{F}_{-}^{\mathrm{H}}(x, y)
$$

where $\mathrm{F}^{\mathrm{H}}(\mathrm{x}, \mathrm{y})$ is called the $\mathrm{H}$-directional derivative of $\mathrm{F}$ in direction $\mathrm{y}$ at $\mathrm{x}$.

By Definition 2.4 and Definition 3.1, we can easily obtain the following Proposition 3.2.

Proposition 3.2. Let $\mathrm{M}$ be an open set in $\mathrm{R}$. If interval-valued function $\mathrm{F}: \mathrm{M} \rightarrow\left([\mathrm{R}], \mathrm{D}_{\mathrm{H}}\right)$ is $\mathrm{H}$-differentiable in the direction $y=1$ at $x^{0} \in M$, then $\mathrm{F}$ is $\mathrm{H}$-differentiable at $x^{0}$, and $\mathrm{F}^{\prime}\left(x^{0}\right)=\mathrm{F}^{\mathrm{H}}\left(x^{0}, 1\right)$.

Theorem 3.3. Let $M$ be an open set in $\mathrm{R}^{\mathrm{n}}$, and $\mathrm{F}: \mathrm{M} \rightarrow\left([\mathrm{R}], \mathrm{D}_{\mathrm{H}}\right)$ be an interval-valued function.

$$
x^{0}=\left(x_{1}^{0}, \cdots, x_{i-1}^{0}, x_{i}^{0}, x_{i+1}^{0}, \cdots, x_{n}^{0}\right) \in M .
$$

If $\mathrm{F}(\mathrm{x})$ is $\mathrm{H}$-differentiable in direction $\mathrm{e}^{\mathrm{i}}(\mathrm{i}=1,2, \cdots, \mathrm{n})$ at $\mathrm{x}^{0}$, then the $\mathrm{i}$-th partial $\mathrm{H}$-derivative of $\mathrm{F}(\mathrm{x})$ at $x^{0}$ exists, and $\frac{\partial \mathrm{F}}{\partial x_{i}}\left(x^{0}\right)=\mathrm{F}^{\mathrm{H}}\left(x^{0}, e^{i}\right)(i=1,2, \cdots, n)$, where

$$
e^{i}=\left(a_{1}^{i}, a_{2}^{i}, \cdots, a_{j}^{i}, \cdots, a_{n}^{i}\right), \text { for } i=1,2, \cdots, n,
$$

with

$$
a_{j}^{i}= \begin{cases}1, & i=j, \\ 0, & i \neq j,\end{cases}
$$

for $\mathrm{j}=1,2, \cdots, \mathrm{n}$. 
Proof. Let $h_{i}\left(x_{i}\right)=F\left(x_{1}^{0}, \cdots, x_{i-1}^{0}, x_{i}, x_{i+1}^{0}, \cdots, x_{n}^{0}\right)$, then

$$
\begin{aligned}
& \frac{h_{i}\left(x_{i}+h\right)-{ }_{H} h_{i}\left(x_{i}\right)}{h}=\frac{F\left(x^{0}+h e^{i}\right)-{ }_{H} F\left(x^{0}\right)}{h}, \\
& \frac{h_{i}\left(x_{i}\right)-{ }_{H} h_{i}\left(x_{i}-h\right)}{h}=\frac{F\left(x^{0}\right)-H F\left(x^{0}-h e^{i}\right)}{h} .
\end{aligned}
$$

Because $F(x)$ is $\mathrm{H}$-differentiable in direction $e^{i}(i=1,2, \cdots, n)$ at $x^{0}$, i.e., there exists $\mathrm{F}^{\mathrm{H}}\left(\chi^{0}, e^{i}\right) \in[R]$ such that

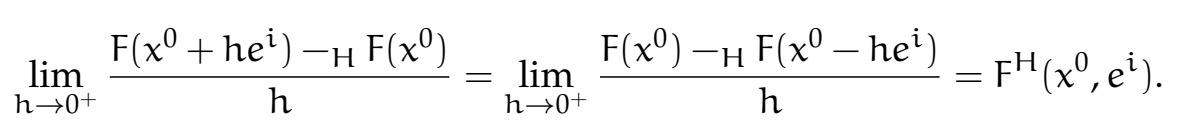

So

$$
\lim _{h \rightarrow 0^{+}} \frac{h_{i}\left(x_{i}+h\right)-H h_{i}\left(x_{i}\right)}{h}=\lim _{h \rightarrow 0^{+}} \frac{h_{i}\left(x_{i}\right)-H h_{i}\left(x_{i}-h\right)}{h}=F^{H}\left(x^{0}, e^{i}\right) .
$$

According to Proposition 3.2, the H-derivative of $h_{i}\left(x_{i}\right)$ at $x_{i}^{0}$ exists. So by Definition 2.5, the $i$-th partial $H$-derivative of $F(x)$ at $x^{0}$ for $x_{i}$ exists, and $\frac{\partial F}{\partial x_{i}}\left(x^{0}\right)=F^{H}\left(x^{0}, e^{i}\right)$.

The following Theorem 3.4 gives a characterization of $\mathrm{H}$-directional differentiability of interval-valued function $\mathrm{F}: M \rightarrow[R]$.

Theorem 3.4. Let $\mathrm{F}: M \rightarrow\left([R], \mathrm{D}_{\mathrm{H}}\right)$ be an interval-valued function, $x \in M, y \in \mathrm{R}^{\mathrm{n}}$. Then

(1) $\mathrm{F}$ is right (resp. left) $\mathrm{H}$-differentiable in direction $\mathrm{y}$ at $\mathrm{x}$, if and only if there exists $\delta>0$ such that

$$
x+h y_{e} \in M\left(x-h y_{e} \in M\right),
$$

and $\mathrm{H}$-difference $\mathrm{F}\left(\mathrm{x}+\mathrm{h} \mathrm{y}_{e}\right)-_{\mathrm{H}} \mathrm{F}(\mathrm{x})\left(\mathrm{F}(\mathrm{x})-_{\mathrm{H}} \mathrm{F}\left(\mathrm{x}-\mathrm{h} \mathrm{y}_{e}\right)\right)$ exists for any $\mathrm{h} \in(0, \delta)$, and there exists $\mathrm{a}_{+} \in$ $[R]\left(a_{-} \in[R]\right)$ such that

$$
\begin{gathered}
\lim _{h \rightarrow 0^{+}} \frac{\underline{F}\left(x+h y_{e}\right)-\underline{F}(x)}{h}=\underline{a}_{+} \text {and } \lim _{h \rightarrow 0^{+}} \frac{\bar{F}\left(x+h y_{e}\right)-\bar{F}(x)}{h}=\bar{a}_{+}, \\
\left(\lim _{h \rightarrow 0^{+}} \frac{F(x)-\underline{F}\left(x-h y_{e}\right)}{h}=\underline{a}_{-} \text {and } \lim _{h \rightarrow 0^{+}} \frac{\bar{F}(x)-H \bar{F}(x-h y)}{h}=\bar{a}_{-}\right) .
\end{gathered}
$$

(2) $\mathrm{F}$ is $\mathrm{H}$-differentiable in the direction $\mathrm{y}$ at $\mathrm{x}$, if and only if there exists $\delta>0$ such that $\mathrm{x}+\mathrm{h} \mathrm{y}_{\mathrm{e}} \in \mathrm{M}$, $x-h y_{e} \in M$, and $\mathrm{H}$-difference

$$
F\left(x+h y_{e}\right)-_{H} F(x), \quad F(x)-_{H} F\left(x-h y_{e}\right),
$$

exists for any $\mathrm{h} \in(0, \delta)$, and there exists $\mathrm{a} \in[\mathrm{R}]$ such that

$$
\begin{aligned}
& \lim _{h \rightarrow 0^{+}} \frac{\overline{\mathrm{F}}\left(x+h y_{e}\right)-\overline{\mathrm{F}}(x)}{h}=\lim _{h \rightarrow 0^{+}} \frac{\overline{\mathrm{F}}(x)-\overline{\mathrm{F}}\left(x-h y_{e}\right)}{h}=\bar{a}, \\
& \lim _{h \rightarrow 0^{+}} \frac{\underline{\mathrm{F}}\left(x+h y_{e}\right)-\underline{F}(x)}{h}=\lim _{h \rightarrow 0^{+}} \frac{\underline{F}(x)-\underline{F}\left(x-h y_{e}\right)}{h}=\underline{a} .
\end{aligned}
$$

Proof. (1) Necessity. Let $\mathrm{F}$ be right $\mathrm{H}$-differentiable in direction $\mathrm{y}$ at $x$, then there exists $\delta>0$ such that $x+h y_{e} \in M$, and $F\left(x+h y_{e}\right)-_{H} F(x)$ exists for any $h \in(0, \delta)$, and there exists $a_{+} \in[R]$ such that

$$
\lim _{h \rightarrow 0^{+}} \frac{F\left(x+h y_{e}\right)-{ }_{H} F(x)}{h}=a_{+} .
$$


By the Hausdorff distance equation and Proposition 2.2 we obtain

$$
\begin{aligned}
\lim _{h \rightarrow 0^{+}} D_{H}\left(\frac{1}{h}\left(F\left(x+h y_{e}\right)-{ }_{H} F(x)\right), a_{+}\right) & =\left(\left|\frac{1}{h}\left(\underline{F}\left(x+h y_{e}\right)-\underline{F}(x)\right)-\underline{a}_{+}\right|,\left|\frac{1}{h}\left(\bar{F}\left(x+h y_{e}\right)-\bar{F}(x)\right)-\bar{a}_{+}\right|\right) \\
& =0 .
\end{aligned}
$$

Therefore, we have

$$
\lim _{h \rightarrow 0^{+}}\left(\frac{1}{h}\left(\underline{F}\left(x+h y_{e}\right)-\underline{F}(x)\right)-\underline{a}_{+}\right)=0, \text { and } \lim _{h \rightarrow 0^{+}}\left(\frac{1}{h}\left(\bar{F}\left(x+h y_{e}\right)-\bar{F}(x)\right)-\bar{a}_{+}\right)=0,
$$

i.e.,

$$
\lim _{h \rightarrow 0^{+}} \frac{\underline{F}\left(x+h y_{e}\right)-\underline{F}(x)}{h}=\underline{a}_{+} \text {, and } \lim _{h \rightarrow 0^{+}} \frac{\bar{F}\left(x+h y_{e}\right)-\bar{F}(x)}{h}=\bar{a}_{+} .
$$

Likewise, $\mathrm{F}$ is left $\mathrm{H}$-differentiable in the direction $\mathrm{y}$ at $\mathrm{x}$, i.e.,

$$
\lim _{h \rightarrow 0^{+}} \frac{F(x)-{ }_{H} F\left(x-h y_{e}\right)}{h}=a_{-} \in[R] .
$$

We can obtain that

$$
\lim _{h \rightarrow 0^{+}} \frac{\underline{F}(x)-\underline{F}(x-h y)}{h}=\underline{a}_{-}, \quad \text { and } \lim _{h \rightarrow 0^{+}} \frac{\bar{F}(x)-\bar{F}(x-h y)}{h}=\bar{a}_{-} .
$$

Sufficiency. Assume that there exists $\delta>0$ such that $x+h y_{e} \in M$, and H-difference $F\left(x+h_{e} y\right)-H F(x)$ exists for any $h \in(0, \delta)$, and there exists $a_{+} \in[R]$ such that

Then we have

$$
\lim _{h \rightarrow 0^{+}} \frac{\underline{F}\left(x+h y_{e}\right)-\underline{F}(x)}{h}=\underline{a}_{+} \text {, and } \lim _{h \rightarrow 0^{+}} \frac{\bar{F}\left(x+h y_{e}\right)-\bar{F}(x)}{h}=\bar{a}_{+} .
$$

$$
\begin{aligned}
\lim _{h \rightarrow 0^{+}} & D_{H}\left(\frac{F\left(x+h y_{e}\right)-{ }_{H} F(x)}{h}, a_{+}\right) \\
& =\lim _{h \rightarrow 0^{+}} \max \left(\left|\frac{\mid \underline{F}\left(x+h y_{e}\right)-\underline{F}(x)}{h}-\underline{a}_{+}\right|,\left|\frac{\bar{F}\left(x+h y_{e}\right)-\bar{F}(x)}{h}-\bar{a}_{+}\right|\right) \\
& =0,
\end{aligned}
$$

i.e.,

$$
\lim _{h \rightarrow 0^{+}} \frac{F\left(x+h y_{e}\right)-H}{h}=a_{+} .
$$

Therefore, $\mathrm{F}$ is right $\mathrm{H}$-diffentiable in the direction $y$ at $x$.

Similarly, we can obtain $\mathrm{F}$ is left $\mathrm{H}$-differentiable in direction $y$ at $x$.

(2) Necessity. Let $F$ be $H$-differentiable in direction $y$ at $x$, then there exists $\delta>0$ such that $x+h y_{e} \in M$, $x-h y_{e} \in M$ and H-differences $F\left(x+h y_{e}\right)-_{H} F(x), F(x)-_{H} F\left(x-h y_{e}\right)$ exist for any $h \in(0, \delta)$, and there exists $a \in[R]$ such that

$$
\lim _{h \rightarrow 0^{+}} \frac{F\left(x+h y_{e}\right)-H F(x)}{h}=\lim _{h \rightarrow 0^{+}} \frac{F(x)-H F\left(x-h y_{e}\right)}{h}=a .
$$

Then we obtain

$$
\begin{aligned}
\lim _{h \rightarrow 0^{+}} & D_{H}\left(\frac{F\left(x+h y_{e}\right)-{ }_{H} F(x)}{h}, a\right) \\
& =\lim _{h \rightarrow 0^{+}} \max \left(\left|\frac{F\left(x+h y_{e}\right)-\underline{F}(x)}{h}-\underline{a}\right|,\left|\frac{\bar{F}\left(x+h y_{e}\right)-\bar{F}(x)}{h}-\bar{a}\right|\right) \\
& =0 .
\end{aligned}
$$


Therefore,

$$
\lim _{h \rightarrow 0^{+}} \frac{\underline{F}\left(x+h y_{e}\right)-\underline{F}(x)}{h}=\underline{a} \text {, and } \lim _{h \rightarrow 0^{+}} \frac{\bar{F}\left(x+h y_{e}\right)-\bar{F}(x)}{h}=\bar{a} .
$$

Likewise, we can obtain

$$
\lim _{h \rightarrow 0^{+}} \frac{\underline{F}(x)-\underline{F}\left(x-h y_{e}\right)}{h}=\underline{a}, \quad \text { and } \quad \lim _{h \rightarrow 0^{+}} \frac{\bar{F}(x)-\bar{F}\left(x-h y_{e}\right)}{h}=\bar{a} .
$$

Moreover, we have

$$
\begin{aligned}
& \lim _{h \rightarrow 0^{+}} \frac{\underline{F}\left(x+h y_{e}\right)-\underline{F}(x)}{h}=\lim _{h \rightarrow 0^{+}} \frac{\underline{F}(x)-\underline{F}\left(x-h y_{e}\right)}{h}=\underline{a}, \\
& \lim _{h \rightarrow 0^{+}} \frac{\bar{F}\left(x+h y_{e}\right)-\bar{F}(x)}{h}=\lim _{h \rightarrow 0^{+}} \frac{\bar{F}(x)-\bar{F}\left(x-h y_{e}\right)}{h}=\bar{a} .
\end{aligned}
$$

Sufficiency. Assume that there exists $\delta>0$ such that $x+h y_{e} \in M, x-h y_{e} \in M$, and H-differences

$$
F\left(x+h_{e} y\right)-{ }_{H} F(x), F(x)-{ }_{H} F\left(x-y_{e}\right),
$$

exist for any $h \in(0, \delta)$, and there exists $a \in[R]$ such that

$$
\begin{aligned}
& \lim _{h \rightarrow 0^{+}} \frac{\bar{F}\left(x+h y_{e}\right)-\bar{F}(x)}{h}=\lim _{h \rightarrow 0^{+}} \frac{\bar{F}(x)-\bar{F}\left(x-h y_{e}\right)}{h}=\bar{a}, \\
& \lim _{h \rightarrow 0^{+}} \frac{\underline{F}\left(x+h y_{e}\right)-\underline{F}(x)}{h}=\lim _{h \rightarrow 0^{+}} \frac{\underline{F}(x)-\underline{F}\left(x-h y_{e}\right)}{h}=\underline{a} .
\end{aligned}
$$

Then we have

$$
\begin{aligned}
\lim _{h \rightarrow 0^{+}} & D_{H}\left(\frac{F\left(x+h y_{e}\right)-{ }_{H} F(x)}{h}, a\right) \\
& =\lim _{h \rightarrow 0^{+}} \max \left(\left|\frac{F\left(x+h y_{e}\right)-\underline{F}(x)}{h}-\underline{a}\right|,\left|\frac{\bar{F}\left(x+h y_{e}\right)-\bar{F}(x)}{h}-\bar{a}\right|\right) \\
& =0 .
\end{aligned}
$$

Therefore,

$$
\lim _{h \rightarrow 0^{+}} \frac{F\left(x+h y_{e}\right)-{ }_{H} F(x)}{h}=a,
$$

i.e.,

$$
\mathrm{F}_{+}^{\mathrm{H}}(\mathrm{x}, \mathrm{y})=\mathrm{a} .
$$

Similarly, we can obtain $\mathrm{F}_{-}^{\mathrm{H}}(x, y)=a$. According to Definition 3.1, $\mathrm{F}$ is $\mathrm{H}$-differentiable in direction $\mathrm{y}$ at $x$.

By Theorem 3.4 and concept of the directional derivative of real-valued function, we can easily obtain the following Corollary 3.5.

Corollary 3.5. Let $\mathrm{F}: \mathrm{M} \rightarrow\left([\mathrm{R}], \mathrm{D}_{\mathrm{H}}\right)$ be an interval-valued function $\mathrm{F}(\mathrm{x})=[\mathrm{F}(\mathrm{x}), \overline{\mathrm{F}}(\mathrm{x})]$. If $\mathrm{F}(\mathrm{x})$ is $\mathrm{H}$-differentiable in direction $y$ at $x^{0}$, then the directional derivatives of $\overline{\mathrm{F}}(\mathrm{x})$ and $\underline{\mathrm{F}}(\mathrm{x})$ in direction $\mathrm{y}$ at $\mathrm{x}^{0}$ exist, and

$$
\underline{F}\left(x^{0}, y\right)=\underline{F^{H}}\left(x^{0}, y\right), \quad \bar{F}\left(x^{0}, y\right)=\overline{F^{H}}\left(x^{0}, y\right) .
$$

We can also obtain the following Corollary 3.6 according to Corollary 3.5 and the uniqueness of directional derivative of the real-valued function.

Corollary 3.6. Let $\mathrm{F}: \mathrm{M} \rightarrow\left([\mathrm{R}], \mathrm{D}_{\mathrm{H}}\right)$ be an interval-valued function. If $\mathrm{F}(\mathrm{x})$ is $\mathrm{H}$-differentiable in the direction $\mathrm{y}$ at $\mathrm{x}$, then the $\mathrm{H}$-directional derivative $\mathrm{F}^{\mathrm{H}}(\mathrm{x}, \mathrm{y})$ of $\mathrm{F}(\mathrm{x})$ is unique. 
Theorem 3.7. Let $\mathrm{F}: \mathrm{M} \rightarrow\left([\mathrm{R}], \mathrm{D}_{\mathrm{H}}\right)$ be an interval-valued function, $\mathrm{x}_{0} \in \mathrm{M}$. Suppose that directional derivative $\underline{\mathrm{F}}\left(\mathrm{x}^{0}, \mathrm{y}\right)$ of $\underline{\mathrm{F}}(\mathrm{x})$ and directional derivative $\overline{\mathrm{F}}\left(\mathrm{x}^{0}, \mathrm{y}\right)$ of $\overline{\mathrm{F}}(\mathrm{x})$ in direction $\mathrm{y}$ at $\mathrm{x}^{0}$ both exist.

(1) If $\underline{\mathrm{F}}\left(\mathrm{x}^{0}, \mathrm{y}\right)<\overline{\mathrm{F}}\left(\mathrm{x}^{0}, \mathrm{y}\right)$, then $\mathrm{F}$ is $\mathrm{H}$-differentiable in the direction $\mathrm{y}$ at $\mathrm{x}^{0}$, and

$$
\mathrm{F}^{\mathrm{H}}\left(x^{0}, y\right)=\left[\underline{\mathrm{F}}\left(x^{0}, y\right), \overline{\mathrm{F}}\left(x^{0}, y\right)\right]
$$

(2) If $\underline{\mathrm{F}}\left(\mathrm{x}^{0}, \mathrm{y}\right)>\overline{\mathrm{F}}\left(\mathrm{x}^{0}, \mathrm{y}\right)$, then $\mathrm{F}$ is $\mathrm{H}$-non-differentiable in the direction $\mathrm{y}$ at $\mathrm{x}^{0}$.

Proof. (1) Suppose that the directional derivatives of $\bar{F}(x)$ and $\underline{F}(x)$ in direction $y$ at $x^{0}$ exist, which is denoted as $\bar{F}\left(x^{0}, y\right)$ and $\underline{F}\left(x^{0}, y\right)$. According to the concept of directional derivative of real-valued functions, we can easily obtain

$$
\begin{aligned}
& \lim _{h \rightarrow 0^{+}} \frac{F\left(x^{0}+h y_{e}\right)-\underline{F}\left(x^{0}\right)}{h}=\lim _{h \rightarrow 0^{+}} \frac{\underline{F}\left(x^{0}\right)-\underline{F}\left(x^{0}-h y_{e}\right)}{h}=\underline{F}\left(x^{0}, y\right), \\
& \lim _{h \rightarrow 0^{+}} \frac{\bar{F}\left(x^{0}+h y_{e}\right)-\bar{F}\left(x^{0}\right)}{h}=\lim _{h \rightarrow 0^{+}} \frac{\bar{F}\left(x^{0}\right)-\bar{F}\left(x^{0}-h y_{e}\right)}{h}=\bar{F}\left(x^{0}, y\right) .
\end{aligned}
$$

On the other hand, from $\underline{F}\left(x^{0}, y\right)<\bar{F}\left(x^{0}, y\right)$, we have $\underline{F}\left(x^{0}, y\right)-\bar{F}\left(x^{0}, y\right)>0$. According to the signpreserving theorem of limit, there exists $\delta>0$ such that $x+h y_{e} \in M, x-h y_{e} \in M$, and

$$
\begin{aligned}
& \underline{F}\left(x^{0}+h y_{e}\right)-\underline{F}\left(x^{0}\right) \leqslant \bar{F}\left(x^{0}+h y_{e}\right)-\bar{F}\left(x^{0}\right), \\
& \underline{F}\left(x^{0}\right)-\underline{F}\left(x^{0}-h y_{e}\right) \leqslant \bar{F}\left(x^{0}\right)-\bar{F}\left(x^{0}-h y_{e}\right),
\end{aligned}
$$

for any $h \in(0, \delta)$, i.e., the $H$-differences $F\left(x+h y_{e}\right)-_{H} F(x)$ and $F(x)-{ }_{H} F\left(x-h y_{e}\right)$ exist.

Let $a\left(x^{0}, y\right)=\left[\underline{F}\left(x^{0}, y\right), \bar{F}\left(x^{0}, y\right)\right]$, then

$$
\begin{aligned}
\lim _{h \rightarrow 0^{+}} & D_{H}\left(\frac{F\left(x^{0}+h y_{e}\right)-H F\left(x^{0}\right)}{h}, a\left(x^{0}, y\right)\right) \\
& =\lim _{h \rightarrow 0^{+}} \max \left(\left|\frac{\underline{F}\left(x^{0}+h y_{e}\right)-\underline{F}\left(x^{0}\right)}{h}-\underline{a}\left(x^{0}, y\right)\right|,\left|\frac{\bar{F}\left(x^{0}+h y_{e}\right)-\bar{F}\left(x^{0}\right)}{h}-\bar{a}\left(x^{0}, y\right)\right|\right) \\
& =0 .
\end{aligned}
$$

Therefore, we have

$$
\lim _{h \rightarrow 0^{+}} \frac{1}{h}\left(F\left(x^{0}+h y_{e}\right)-{ }_{H} F\left(x^{0}\right)\right)=a\left(x^{0}, y\right) .
$$

So $F_{+}^{\mathrm{H}}\left(x^{0}, y\right)=a\left(x^{0}, y\right)$.

The proof of $\mathrm{F}_{-}^{\mathrm{H}}\left(x^{0}, y\right)=a\left(x^{0}, y\right)$ can be completed with similar argument. So $F(x)$ is H-differentiable in direction $y$ at $x^{0}$, and

$$
\mathrm{F}^{\mathrm{H}}\left(x^{0}, y\right)=\left[\underline{\mathrm{F}}\left(x^{0}, y\right), \overline{\mathrm{F}}\left(x^{0}, y\right)\right] .
$$

(2) Assume that $F(x)$ is $\mathrm{H}$-differentiable in the direction $y$ at $x_{0}$, with $\mathrm{H}$-directional derivative

$$
\mathrm{F}^{\mathrm{H}}\left(x^{0}, y\right)=\left[\underline{\mathrm{F}^{\mathrm{H}}}\left(x^{0}, y\right), \overline{\mathrm{FH}^{\mathrm{H}}}\left(x^{0}, y\right)\right] \in[R] .
$$

From Corollary 3.5, we have

$$
\overline{\mathrm{F}^{\mathrm{H}}}\left(x^{0}, y\right)=\overline{\mathrm{F}}\left(x^{0}, y\right) \text {, and } \underline{\mathrm{F}^{\mathrm{H}}}\left(x^{0}, y\right)=\underline{\mathrm{F}}\left(x^{0}, y\right) .
$$

By Corollary 3.6, this shows that

$$
\mathrm{F}^{\mathrm{H}}\left(x^{0}, y\right)=\left[\underline{F}\left(x^{0}, y\right), \overline{\mathrm{F}}\left(x^{0}, y\right)\right] .
$$

So $\underline{F}\left(x^{0}, y\right) \leqslant \bar{F}\left(x^{0}, y\right)$, which contradicts the hypothesis $\underline{F}\left(x^{0}, y\right)>\bar{F}\left(x^{0}, y\right)$. 
Example 3.8. Let $M=(0,3) \times(0,5)$, and

$$
F(x)=\left[\left(x_{1}+x_{2}\right)^{2}+1,\left(x_{1}+x_{2}\right)^{2}+3\right], \quad x \in M,
$$

be an interval-valued function defined on $M$.

For point $x_{0}=\left(x_{1}^{0}, x_{2}^{0}\right) \in(0,3) \times(0,5)$ in direction $y=\left(y_{1}, y_{2}\right) \in[0,+\infty) \times[0,+\infty),\left(y_{e}=\left(y_{1 e}, y_{2 e}\right)\right.$ is a unit vector of $\left.y=\left(y_{1}, y_{2}\right)\right)$, we have

$$
\begin{aligned}
\underline{F}_{+}\left(x^{0}, y\right) & =\lim _{h \rightarrow 0^{+}} \frac{\underline{F}\left(x^{0}+h y_{e}\right)-\underline{F}\left(x^{0}\right)}{h} \\
& =\lim _{h \rightarrow 0^{+}} \frac{\left[x_{1}^{0}+x_{2}^{0}+h\left(y_{1 e}+y_{2 e}\right)\right]^{2}+1-\left[\left(x_{1}^{0}+x_{2}^{0}\right)^{2}+1\right]}{h} \\
& =\lim _{h \rightarrow 0^{+}} \frac{2 h\left(y_{1 e}+y_{2 e}\right)\left(x_{1}^{0}+x_{2}^{0}\right)+h^{2}\left(y_{1 e}+y_{2 e}\right)^{2}}{h} \\
& =2\left(x_{1}^{0}+x_{2}^{0}\right)\left(y_{1 e}+y_{2 e}\right), \\
\underline{F}_{-}\left(x^{0}, y\right) & =\lim _{h \rightarrow 0^{+}} \frac{\underline{F}\left(x^{0}\right)-\underline{F}\left(x^{0}-h y_{e}\right)}{h} \\
& =\lim _{h \rightarrow 0^{+}} \frac{\left[\left(x_{1}^{0}+x_{2}^{0}\right)^{2}+1\right]-\left[x_{1}^{0}+x_{2}^{0}-h\left(y_{1 e}+y_{2 e}\right)\right]^{2}+1}{h} \\
& =\lim _{h \rightarrow 0^{+}} \frac{2 h\left(x_{1}^{0}+x_{2}^{0}\right)\left(y_{1 e}+y_{2 e}\right)-h^{2}\left(y_{1 e}+y_{2 e}\right)^{2}}{h} \\
& =2\left(x_{1}^{0}+x_{2}^{0}\right)\left(y_{1 e}+y_{2 e}\right) .
\end{aligned}
$$

Therefore, we have

$$
\underline{\mathrm{F}}\left(x^{0}, \mathrm{y}\right)=2\left(x_{1}^{0}+x_{2}^{0}\right)\left(y_{1 e}+y_{2 e}\right)
$$

On the other hand,

$$
\begin{aligned}
\bar{F}_{+}\left(x^{0}, y\right) & =\lim _{h \rightarrow 0^{+}} \frac{\bar{F}\left(x^{0}+h y_{e}\right)-\bar{F}\left(x^{0}\right)}{h} \\
& =\lim _{h \rightarrow 0^{+}} \frac{\left[x_{1}^{0}+x_{2}^{0}+h\left(y_{1 e}+y_{2 e}\right)+1\right]^{2}+3-\left(x_{1}^{0}+x_{2}^{0}+1\right)^{2}-3}{h} \\
& =\lim _{h \rightarrow 0^{+}} \frac{2\left(x_{1}^{0}+x_{2}^{0}+1\right) h\left(y_{1 e}+y_{2 e}\right)+h^{2}\left(y_{1 e}+y_{2 e}\right)^{2}}{h} \\
& =2\left(x_{1}^{0}+x_{2}^{0}+1\right)\left(y_{1 e}+y_{2 e}\right) .
\end{aligned}
$$

Similarly, we have $\underline{F}_{-}\left(x^{0}, y\right)=2\left(x_{1}^{0}+x_{2}^{0}+1\right)\left(y_{1 e}+y_{2 e}\right)$. Hence, we have

$$
\overline{\mathrm{F}}\left(x_{0}, y\right)=2\left(x_{1}^{0}+x_{2}^{0}+1\right)\left(y_{1}+y_{2}\right) \text {. }
$$

So $\underline{F}\left(x^{0}, y\right)<\bar{F}\left(x^{0}, y\right)$. By Theorem 3.7 (1), we have

$$
\mathrm{F}^{\mathrm{H}}\left(x^{0}, y\right)=\left[2\left(x_{1}^{0}+x_{2}^{0}\right)\left(y_{1 e}+y_{2 e}\right), 2\left(x_{1}^{0}+x_{2}^{0}+1\right)\left(y_{1 e}+y_{2 e}\right)\right] .
$$

Example 3.9. Let $M=[0,2] \times[0,2]$, and

$$
F(x)=\left[\left(x_{1}+x_{2}\right)^{2}+x_{1}+x_{2}+1,\left(x_{1}+x_{2}\right)^{2}+3\right], \quad x \in M,
$$

be an interval-valued function defined on $M$.

For $x^{0}=\left(x_{1}^{0}, x_{2}^{0}\right) \in[0,2] \times[0,2]$ in direction $y=\left(y_{1}, y_{2}\right) \in[0,+\infty) \times[0,+\infty)\left(y_{e}=\left(y_{1 e}, y_{2 e}\right)\right.$ is a unit 
vector of $\left.y=\left(y_{1}, y_{2}\right)\right)$, we have

$$
\begin{aligned}
\underline{F}_{+}\left(x^{0}, y\right) & =\lim _{h \rightarrow 0^{+}} \frac{\left[x_{1}^{0}+x_{2}^{0}+h\left(y_{1 e}+y_{2 e}\right)\right]^{2}+\left[x_{1}^{0}+x_{2}^{0}+h\left(y_{1 e}+y_{2 e}\right)\right]+1-\left[\left(x_{1}^{0}+x_{2}^{0}\right)^{2}+\left(x_{1}^{0}+x_{2}^{0}\right)+1\right]}{h} \\
& =\lim _{h \rightarrow 0^{+}} \frac{2\left(x_{1}^{0}+x_{2}^{0}\right) h\left(y_{1 e}+y_{2 e}\right)+h^{2}\left(y_{1 e}+y_{2 e}\right)^{2}+h\left(y_{1 e}+y_{2 e}\right)}{h} \\
& =2\left(x_{1}^{0}+x_{2}^{0}\right)\left(y_{1 e}+y_{2 e}\right)+\left(y_{1 e}+y_{2 e}\right) \\
& =\left[2\left(x_{1}^{0}+x_{2}^{0}\right)+1\right]\left(y_{1 e}+y_{2 e}\right) .
\end{aligned}
$$

Likewise, we can obtain

$$
\underline{F}_{-}\left(x^{0}, y\right)=\left[2\left(x_{1}^{0}+x_{2}^{0}\right)+1\right]\left(y_{1 e}+y_{2 e}\right) .
$$

Therefore,

$$
\underline{\mathrm{F}}\left(\mathrm{x}^{0}, \mathrm{y}\right)=\left(2\left(\mathrm{x}_{1}^{0}+\mathrm{x}_{2}^{0}\right)+1\right)\left(\mathrm{y}_{1 e}+\mathrm{y}_{2 e}\right) .
$$

Similarly, we can obtain $\overline{\mathrm{F}}\left(x^{0}, y\right)=2\left(x_{1}^{0}+x_{2}^{0}\right)\left(y_{1 e}+y_{2 e}\right)$. So $\underline{F}\left(x^{0}, y\right)>\bar{F}\left(x^{0}, y\right)$. By Theorem 3.7 (2), $F$ is $\mathrm{H}$-non-differentiable in direction $y$ at $x^{0}$.

\section{D-direction differentiability of interval-valued function}

In order to avoid the appearance of the difficulty that $\mathrm{H}$-differences bring in the conditions of Definition 3.1, in this section, we introduce the D-directional derivative and D-directional differentiability of interval-valued function from a subset of space $R^{n}$ into interval numbers space $[R]$.

Definition 4.1. Let $F: M \rightarrow\left([R], D_{H}\right)$ be an interval-valued function, $x \in M$. If for $y \in R^{n}$, there exists $\delta>0$ such that $x+h y_{e} \in M\left(x-h y_{e} \in M\right)$ for any $h \in(0, \delta)$, and there exists $a_{+} \in[R]\left(a_{-} \in[R]\right)$ such that

$$
\lim _{h \rightarrow 0^{+}} \frac{D_{H}\left(F\left(x+h y_{e}\right), F(x)+h a_{+}\right)}{h}=0\left(\lim _{h \rightarrow 0^{+}} \frac{D_{H}\left(F(x), F\left(x-h y_{e}\right)+h a_{-}\right)}{h}=0\right) .
$$

Then we say that $F$ is right (resp. left) D-differentiable in direction $y$ at $x$, and call $a_{+}\left(a_{-}\right)$the right (resp. left) $\mathrm{D}$-directional derivative of $\mathrm{F}$ in direction $\mathrm{y}$ at $x$, which is denoted as

$$
F_{+}^{D}(x, y)=a_{+}\left(F_{-}^{D}(x, y)=a_{-}\right) .
$$

If $F_{+}^{D}(x, y)=F_{-}^{D}(x, y)$, we say that $F$ is $D$-differentiable in direction $y$ at $x$, which is denoted as

$$
F^{D}(x, y)=F_{+}^{D}(x, y)=F_{-}^{D}(x, y),
$$

and we call $\mathrm{F}^{\mathrm{D}}(\mathrm{x}, \mathrm{y})$ is the $\mathrm{D}$-directional derivative of $\mathrm{F}$ in direction $\mathrm{y}$ at $\mathrm{x}$.

Theorem 4.2. Let $\mathrm{F}: \mathrm{M} \rightarrow\left([\mathrm{R}], \mathrm{D}_{\mathrm{H}}\right)$ be an interval-valued function, $\mathrm{x} \in \mathrm{M}, \mathrm{y} \in \mathrm{R}^{\mathrm{n}}$. If $\mathrm{F}$ is $\mathrm{D}$-differentiable in direction $y$ at $x$, then $\underline{F}(x)$ and $\bar{F}(x)$ are differentiable in direction $y$ at $x$, and $\mathrm{F}^{\mathrm{D}}(x, y)=[\underline{F}(x, y), \bar{F}(x, y)]$. Conversely, if $\underline{F}(x)$ and $\overline{\mathrm{F}}(\mathrm{x})$ are differentiable in direction $y$ at $\mathrm{x}$, and $\underline{\mathrm{F}}(\mathrm{x}, \mathrm{y}) \leqslant \overline{\mathrm{F}}(\mathrm{x}, \mathrm{y})$. Then $\mathrm{F}$ is $\mathrm{D}$-differentiable in direction $\mathrm{y}$ at $\mathrm{x}$, and

$$
\mathrm{F}^{\mathrm{D}}(x, y)=[\mathrm{F}(x, y), \overline{\mathrm{F}}(x, y)],
$$

where $\underline{F}(x, y)$ and $\bar{F}(x, y)$ are directional derivatives of $\underline{F}(x)$ and $\bar{F}(x)$ in direction $y$ at $x$, respectively.

Proof. Let $F: M \rightarrow\left([R], D_{H}\right)$ be an interval-valued function, which is D-differentiable in direction $y$ at $x$. Then there exists $\delta>0$ such that $x+h y_{e} \in M$ and $x-h y_{e} \in M$ for any $h \in(0, \delta)$, and there exists $\mathrm{F}^{\mathrm{D}}(\mathrm{x}, \mathrm{y}) \in[\mathrm{R}]$ such that

$$
\lim _{h \rightarrow 0^{+}} \frac{D_{H}\left(F\left(x+h y_{e}\right), F(x)+h F^{D}(x, y)\right)}{h}=\lim _{h \rightarrow 0^{+}} \frac{D_{H}\left(F(x), F\left(x-h y_{e}\right)+h F^{D}(x, y)\right)}{h}=0 .
$$


Therefore, we can obtain

$$
\begin{aligned}
\lim _{h \rightarrow 0^{+}} & \frac{D_{H}\left(F\left(x+h y_{e}\right), F(x)+h F^{D}(x, y)\right)}{h} \\
& =\lim _{h \rightarrow 0^{+}} \frac{\max \left\{\mid \underline{F}\left(x+h y_{e}\right)-\left(\frac{F(x)+h F^{D}(x, y)}{h}|,| \bar{F}\left(x+h y_{e}\right)-\left(\overline{F(x)+h F^{D}(x, y)}\right) \mid\right\}\right.}{h} \\
& =\lim _{h \rightarrow 0^{+}} \max \left\{\left|\frac{F\left(x+h y_{e}\right)-\underline{F}(x)-h \underline{F^{D}}(x, y)}{h}\right|,\left|\frac{\bar{F}\left(x+h y_{e}\right)-\bar{F}(x)-h \overline{F^{D}}(x, y)}{h}\right|\right\} \\
& =0 .
\end{aligned}
$$

So

$$
\lim _{h \rightarrow 0^{+}}\left|\frac{F\left(x+h y_{e}\right)-\underline{F}(x)}{h}-\underline{F^{D}}(x, y)\right|=\lim _{h \rightarrow 0^{+}}\left|\frac{\bar{F}\left(x+h y_{e}\right)-\bar{F}(x)}{h}-\overline{F^{D}}(x, y)\right|=0 .
$$

Therefore, the right derivative of $\underline{F}(x)$ and $\bar{F}(x)$ in direction $y$ at $x$ exist, and

$$
\underline{F}_{+}(x, y)=\underline{F^{D}}(x, y), \quad \bar{F}_{+}(x, y)=\overline{F^{D}}(x, y) .
$$

Likewise, from $\lim _{h \rightarrow 0^{+}} \frac{D_{H}\left(F(x), F\left(x-h y_{e}\right)+h F^{D}(x, y)\right)}{h}=0$, we can obtain $\underline{F}(x)$ and $\bar{F}(x)$ is left differentiable in direction $y$ at $x$, and

$$
\underline{F}_{-}(x, y)=\underline{F^{D}}(x, y), \quad \bar{F}_{-}(x, y)=\overline{F^{D}}(x, y) .
$$

Therefore,

$$
\underline{F}_{-}(x, y)=\underline{F}_{+}(x, y)=\underline{F^{D}}(x, y), \quad \bar{F}_{-}(x, y)=\bar{F}_{+}(x, y)=\overline{F^{D}}(x, y) .
$$

So the directional derivatives $\underline{F}(x, y)$ and $\bar{F}(x, y)$ of $\underline{F}(x)$ and $\bar{F}(x)$ in direction $y$ at $x$ exist, and

$$
\mathrm{F}^{\mathrm{D}}(x, y)=[\mathrm{F}(x, y), \overline{\mathrm{F}}(x, y)] \text {. }
$$

Conversely, let the directional derivatives of $\underline{F}(x)$ and $\bar{F}(x)$ in direction $y$ at $x$ exist and $\underline{F}(x, y) \leqslant \bar{F}(x, y)$. According to the concept of directional derivative of real-valued function, there exists $\delta>0$ such that $x+h y_{e} \in M, x-h y_{e} \in M$ for any $h \in(0, \delta)$ and

$$
\lim _{h \rightarrow 0^{+}} \frac{F(x)-\underline{F}\left(x-h y_{e}\right)}{h}=\underline{F}(x, y), \quad \lim _{h \rightarrow 0^{+}} \frac{\bar{F}\left(x+h y_{e}\right)-\bar{F}(x)}{h}=\bar{F}(x, y) .
$$

Let $a(x, y)=[\underline{F}(x, y), \bar{F}(x, y)]$, then $a(x, y) \in[R]$, and we can obtain

$$
\lim _{h \rightarrow 0^{+}} \frac{D_{H}\left(F(x), F\left(x-h y_{e}\right)+h a(x, y)\right)}{h}=0, \quad \lim _{h \rightarrow 0^{+}} \frac{D_{H}\left(F\left(x+h y_{e}\right), F(x)+h a(x, y)\right)}{h}=0 .
$$

Therefore, by Definition 4.1, we obtain $F(x)$ is D-differentiable in direction $y$ at $x$ and

$$
\mathrm{F}^{\mathrm{D}}(x, y)=[\mathrm{F}(x, y), \overline{\mathrm{F}}(x, y)]
$$

The following Theorem 4.3 and Example 4.4 address the relation between D-directional differentiability and H-directional differentiability.

Theorem 4.3. Let $\mathrm{F}: \mathrm{M} \rightarrow\left([\mathrm{R}], \mathrm{D}_{\mathrm{H}}\right)$ be an interval-valued function, $\mathrm{x} \in \mathrm{M}, \mathrm{y} \in \mathrm{R}^{\mathrm{n}}$. If $\mathrm{F}$ is $\mathrm{H}$-differentiable in direction $\mathrm{y}$ at $\mathrm{x}$, then $\mathrm{F}$ is $\mathrm{D}$-differentiable in direction $\mathrm{y}$ at $\mathrm{x}$, and

$$
\mathrm{F}^{\mathrm{D}}(x, y)=\mathrm{F}^{\mathrm{H}}(x, y) \text {. }
$$


Proof. Let $\mathrm{F}$ be $\mathrm{H}$-differentiable in direction $\mathrm{y}$ at $\mathrm{x}$, then there exists $\delta>0$ such that $x+$ hye $\in M$, $x-h y_{e} \in M$ and $F\left(x+h y_{e}\right)-_{H} F(x), F(x)-_{H} F\left(x-h y_{e}\right)$ both exist for any $h \in(0, \delta)$ and there exists $\mathrm{F}^{\mathrm{H}}(\mathrm{x}, \mathrm{y}) \in[\mathrm{R}]$ such that

$$
\lim _{h \rightarrow 0^{+}} \frac{F\left(x+h y_{e}\right)-{ }_{H} F(x)}{h}=\lim _{h \rightarrow 0^{+}} \frac{F(x)-{ }_{H} F\left(x-h y_{e}\right)}{h}=F^{H}(x, y) .
$$

According to Proposition 2.3, we have

$$
\begin{aligned}
\lim _{h \rightarrow 0^{+}} \frac{D_{H}\left(F(x+h y e), F(x)+h F^{H}(x, y)\right)}{h} & =\lim _{h \rightarrow 0^{+}} \frac{D_{H}\left(F(x+h y)-{ }_{H} F(x), h F^{H}(x, y)\right)}{h} \\
& =\lim _{h \rightarrow 0^{+}} D_{H}\left(\frac{F(x+h y)-{ }_{H} F(x)}{h}, F^{H}(x, y)\right) \\
& =0 .
\end{aligned}
$$

So by Definition 4.1, $\mathrm{F}$ is right $\mathrm{D}$-differentiable in direction $\mathrm{y}$ at $\mathrm{x}$, and

$$
F_{+}^{D}(x, y)=F^{H}(x, y) .
$$

Likewise, according to $\lim _{h \rightarrow 0^{+}} \frac{F(x)-H F\left(x-h y_{e}\right)}{h}=F^{H}(x, y)$, we can obtain $F$ is left D-differentiable in direction $y$ at $x$ and

$$
F_{-}^{D}(x, y)=F^{H}(x, y)
$$

Therefore,

$$
F_{-}^{\mathrm{D}}(x, y)=F_{+}^{\mathrm{D}}(x, y)=\mathrm{F}^{\mathrm{H}}(x, y) .
$$

By Definition 4.1, we obtain F is D-differentiable in direction $y$ at $x$, and

$$
F^{D}(x, y)=F^{H}(x, y)
$$

By Theorem 4.3, we know that $\mathrm{F}$ is $\mathrm{H}$-differentiable in direction $\mathrm{y}$ at $\mathrm{x}$, which implies $\mathrm{F}$ is $\mathrm{D}$-differentiable in direction $y$ at $x$. But the following Example 4.4 can show that D-differentiability of $F$ does not mean it is $\mathrm{H}$-differentiable in direction $y$ at $x$.

Example 4.4. Let $F(x)=\left[x^{2}, x^{3}+4\right]$ be an interval-valued function defined on $[-1,1]$. Then for $x=0 \in$ $[-1,1]$ in direction $y=1$, we have

$$
\underline{\mathrm{F}}_{+}(0,1)=\lim _{\mathrm{h} \rightarrow 0^{+}}=\frac{\underline{\mathrm{F}}(0+\mathrm{h}, 1)-\underline{\mathrm{F}}(0)}{\mathrm{h}^{2}}=\lim _{\mathrm{h} \rightarrow 0^{+}} \frac{\mathrm{h}^{2}-0^{2}}{\mathrm{~h}}=0 .
$$

Likewise, we obtain $\underline{F}_{-}(0,1)=\overline{\mathrm{F}}_{+}(0,1)=\overline{\mathrm{F}}_{-}(0,1)=0$. Let $\mathrm{a}(0,1)=0=[0,0]$, then

$$
\begin{aligned}
F_{+}^{D}(0,1) & =\lim _{h \rightarrow 0^{+}} \frac{D_{H}\left(F(0+h, 1), F(0)+h F_{+}(0,1)\right)}{h} \\
& =\lim _{h \rightarrow 0^{+}} \frac{D_{H}\left(\left[h^{2}, h^{3}+4\right],[0,4]\right)}{h} \\
& =\lim _{h \rightarrow 0^{+}} \frac{\max \left\{\left|h^{2}-0\right|,\left|h^{3}+4-4\right|\right\}}{h} \\
& =0 .
\end{aligned}
$$

Similarly, we obtain $\mathrm{F}_{-}^{\mathrm{D}}(0,1)=0$. Therefore, $\mathrm{F}_{\text {is }} \mathrm{D}$-differentiable in direction $\mathrm{y}=1$ at $\mathrm{x}=0$, and

$$
\mathrm{F}^{\mathrm{D}}(0,1)=0=[0,0]
$$


Next we will prove that $F$ is not right $\mathrm{H}$-differentiable in direction $y=1$ at $x=0$.

Suppose that $F$ is right $\mathrm{H}$-differentiable in direction $y=1$ at $x=0$, then there exists $\delta>0$ such that $F(0+h)-H F(0)$ exists, i.e.,

$$
F(0+h)-_{H} F(0)=\left[\underline{F(0+h)-{ }_{H} F(0)}, \overline{F(0+h)-{ }_{H} F(0)}\right] \in[R],
$$

for any $h \in(0, \delta)$. Therefore,

$$
\underline{F(0+h)-_{H} F(0)} \leqslant \overline{F(0+h)-{ }_{H} F(0)} .
$$

By Propsition 2.2, we have

$$
\underline{F(0+h)}-\underline{F(0)} \leqslant \overline{\bar{F}(0+h)}-\overline{F(0)}
$$

i.e.,

$$
(0+h)^{2}-(0)^{2} \leqslant(0+h)^{3}+4-\left(0^{3}+4\right) .
$$

So $h^{2} \leqslant h^{3}$ for any $h \in(0, \delta)$, i.e., $h \geqslant 1$. Thus we can obtain a contradictory conclusion that $h \geqslant 1$ for any $h \in(0, \delta)$. So $F$ is not right $\mathrm{H}$-differentiable in direction $y=1$ at $x=0$. According to Definition 3.1, it is not $\mathrm{H}$-differentiable in direction $\mathrm{y}=1$ at $x=0$.

In what follows, we will further give a sufficient and necessary condition such that D-directional differentiable interval-valued function $\mathrm{F}: M \rightarrow\left([R], \mathrm{D}_{\mathrm{H}}\right)$ becomes $\mathrm{H}$-directional differentiable.

Theorem 4.5. Let $\mathrm{F}: M \rightarrow\left([\mathrm{R}], \mathrm{D}_{\mathrm{H}}\right)$ be an interval-valued function, $\mathrm{x} \in \mathrm{M}, \mathrm{y} \in \mathrm{R}^{\mathrm{n}}$. $\mathrm{F}$ is $\mathrm{H}$-differentiable in direction $\mathrm{y}$ at $\mathrm{x}$, if and only if $\mathrm{F}$ is $\mathrm{D}$-differentiable in direction $\mathrm{y}$ at $\mathrm{x}$, and there exists $\delta>0$ such that $\mathrm{H}$-differences $\mathrm{F}\left(\mathrm{x}+\mathrm{h} \mathrm{y}_{\mathrm{e}}\right)-_{\mathrm{H}} \mathrm{F}(\mathrm{x})$ and $\mathrm{F}(\mathrm{x})-_{\mathrm{H}} \mathrm{F}\left(\mathrm{x}-\mathrm{h} \mathrm{y}_{\mathrm{e}}\right)$ exist for any $\mathrm{h} \in(0, \delta)$.

Proof. Necessary. Suppose that $\mathrm{F}$ is $\mathrm{H}$-differentiable in direction $\mathrm{y}$ at $\mathrm{x}$, then according to Definition 3.1, there exists $\delta>0$ such that $H$-differences $F\left(x+h y_{e}\right)-_{H} F(x)$ and $F(x)-{ }_{H} F\left(x-h y_{e}\right)$ exist for any $h \in(0, \delta)$.

On the other hand, according to Theorem 4.3, we can infer that F is D-differentiable in direction $y$ at $x$.

Sufficiency. Suppose that $F$ is D-differentiable in direction $y$ at $x$. Then there exists $\delta_{1}>0$ such that $x+h y_{e} \in M, x-h y_{e} \in M$ for any $h \in\left(0, \delta_{1}\right)$ and there exists $F^{D}(x, y) \in[R]$ such that

$$
\lim _{h \rightarrow 0^{+}} \frac{D_{H}\left(F\left(x+h y_{e}\right), F(x)+h F^{D}(x, y)\right)}{h}=\lim _{h \rightarrow 0^{+}} \frac{D_{H}\left(F(x), F\left(x-h y_{e}\right)+h F^{D}(x, y)\right)}{h}=0 .
$$

Take $\delta_{0}=\min \left\{\delta, \delta_{1}\right\}$, then $x+h y_{e} \in M, x-h y_{e} \in M$ and H-differences

$$
F\left(x+h y_{e}\right)-{ }_{H} F(x), \quad F(x)-{ }_{H} F\left(x-h y_{e}\right),
$$

exist for any $h \in\left(0, \delta_{0}\right)$.

According to Proposition 2.3, we have

$$
\begin{aligned}
\lim _{h \rightarrow 0^{+}} D_{H}\left(\frac{F\left(x+h y_{e}\right)-{ }_{H} F(x)}{h}, F^{D}(x, y)\right) & =\lim _{h \rightarrow 0^{+}} \frac{D_{H}\left(F\left(x+h y_{e}\right)-H F(x), h F^{D}(x, y)\right)}{h} \\
& =\lim _{h \rightarrow 0^{+}} \frac{D_{H}\left(F\left(x+h y_{e}\right), F(x)+h F^{D}(x, y)\right)}{h} \\
& =0 .
\end{aligned}
$$

So by Definition 3.1, $F$ is H-differentiable in direction $y$ at $x$, and $F_{+}^{H}(x, y)=F^{D}(x, y)$.

Similarly, we can obtain $F(x)$ is left $H$-differentiable in direction $y$ at $x$, and $F_{-}^{\mathrm{H}}(x, y)=\mathrm{F}^{\mathrm{D}}(x, y)$. So according to Definition 3.1, $\mathrm{F}$ is $\mathrm{H}$-differentiable in direction $\mathrm{y}$ at $\mathrm{x}$.

\section{Conclusion}

Reference $[7,8]$ give the concepts of the $\mathrm{H}$-derivative of the interval-valued function from nonempty subset of real-valued space to interval-valued space and the partial $\mathrm{H}$-derivative of the interval-valued function from nonempty subset of $n$-dimensional Euclidean space to interval number space by using 
H-difference which only take the changing rate of interval-valued function in axis direction into consideration. In this paper, we take the changing rate of interval-valued function in special direction into consideration, and give the concepts of the $\mathrm{H}$-directional derivative of the interval-valued function from nonempty subset of $n$-dimensional Euclidean space to interval number space, and prove that $\mathrm{H}$-derivative and partial $\mathrm{H}$-derivative are both $\mathrm{H}$-derivatives of interval-valued function in axis direction.

We know that the H-difference between two interval numbers does not always exist, which makes it difficult to study the differentiability of interval-valued function. In order to solve this problem, we give the concept of D-directional derivative of interval-valued functions from nonempty subset of $n$ dimensional Euclidean space to interval number space by using Hausdorff distance in Section 4 and we prove that an interval-valued function which is $\mathrm{H}$-differentiable must be $\mathrm{D}$-differentiable, but the opposite is not always true.

The concept of D-differentiability expands the species of the interval-valued function which is differentiable. This will enrich and develop the theory and application of the differentiability of interval-valued function. We hope it can provide a new method of studying the problem about the theory of differentiability of interval-valued function and its application.

\section{Acknowledgment}

The authors express their sincere gratitude to the editors and anonymous referees for useful comments that helped to improve the presentation of the results and accentuate important details. This research is supported by the National Science Fund of China (11461052) and the Inner Mongolia Natural Science Foundation of China (2014MS0107).

\section{References}

[1] Y. Chalco-Cano, H. Román-Flores, M. D. Jiménez-Gamero, Generalized derivative and $\pi$-derivative for set-valued functions, Inform. Sci., 181 (2011), 2177-2188. 1

[2] Y. Chalco-Cano, A. Rufián-Lizan, H. Román-Flores, M. D. Jiménez-Gamero, Calculus for interval-valued functions using generalized Hukuhara derivative and applications, Fuzzy Sets and Systems, 219 (2013), 49-67. 1

[3] V. Lupulescu, Fractional calculus for interval-valued functions, Fuzzy Sets and Systems, 265 (2015), 63-85. 1

[4] R. E. Moore, Interval Analysis, Prentice-Hall, New Jersey, (1966). 1

[5] R. Osuna-Gómez, Y. Chalco-Cano, B. Hernández-Jiménez, G. Ruiz-Garzón, Optimality conditions for generalized differentiable interval-valued functions, Inform. Sci., 321 (2015), 136-146. 1

[6] L. Stefanini, B. Bede, Generalized Hukuhara differentiability of interval-valued functions and interval differential equations, Nonlinear Anal., 71 (2009), 1311-1328. 1

[7] H.-C. Wu, The Karush-Kuhn-Tucker optimality conditions in an optimization problem with interval-valued objective functions, European J. Oper. Res., 176 (2007), 46-59. 1, 2.1, 2.4, 2.5, 3, 5

[8] H.-C. Wu, The Karush-Kuhn-Tucker optimality conditions in multiobjective programming problems with interval-valued objective functions, European J. Oper. Res., 196 (2009), 49-60. 1, 3, 5 